\title{
Visitador médico virtual: una ventana estratégica para el desarrollo del mercado farmacéutico
}

\author{
José Eustaquio Palma Rojas ${ }^{1}$ \\ Marelbis Xiomara Arregocés Vanegas ${ }^{2}$
}

\section{Resumen}

Una visita médica es exitosa en la medida en que el visitador consiga que el médico adopte los productos que su laboratorio promociona y ofrece.

Teniendo en cuenta que los adelantos tecnológicos del mundo actual permiten el acceso a información relacionada con diversidad de productos y servicios, surge la posibilidad de adoptar la figura del visitador médico virtual, que facilite a la comunidad de facultativos acceder tanto al conocimiento de nuevos productos como a la actualización de los ya existentes.

Esta investigación, la cual se enmarca dentro del área temática de Estado, empresa, globalización y desarrollo, busca determinar las características ideales de una combinación de servicios en línea y atención personalizada, que contribuya a una eficacia operacional de los laboratorios farmacéuticos, de tal manera que permita aumentar su cobertura, disminuir los gastos de mercadeo y capacitar a los visitadores médicos en las estrategias para el desarrollo del mercado.

El estudio, realizado en Barranquilla y Riohacha, mediante la encuesta aplicada a 133 médicos, es de carácter cuantitativo-descriptivo-exploratorio, con proyección a la implementación de la visita médica virtual como estrategia para promover y desarrollar el mercado farmacéutico.

Palabras clave: Desarrollo del mercado, valoración del servicio de visita médica, ventana estratégica, uso del servicio, satisfacción general, satisfacción de atributos.

\section{Abstract}

A medical details is successful to the extent that visitor gets the doctor takes the products that his laboratory promoted and offered. Given that technological advances around the world provide access to information related to diversity of products and services, there is the possibility of adopting the figure of the virtual health visitor to provide the community of doctors access to the knowledge of new products and updating existing ones. This research, inside of subject area globalization and development company, seeks to determine the ideal characteristics of a combination of online services and personal attention, that contributes to operational effectiveness of the drug companies, that increase the coverage, low marketing expenses and training to medical strategies for market development. The study, conducted in the cities of Barranquilla and Riohacha by means of survey to 133 doctors, is a quantitative-descriptiveexploratory study, projected to the implementation of the virtual medical detail as a strategy for the promotion and development of the pharmaceutical market.

Key words: Market development, rating service of medical detail, strategic window, use of the service, overall satisfaction, satisfaction attributes.

Recibido: 16 de abril de 2012

Aprobado: 27 de julio de 2012

\footnotetext{
${ }^{1}$ Administrador de empresas. Tutor, UNAD-Barranquilla, jose.palma@unad.edu.co

2 Ingeniera industrial. Consejera, UNAD-Riohacha, marelvis.arregoces@unad.edu.co
} 


\section{Introducción}

La visita médica, estrategia utilizada por organizaciones del sector farmacéutico para promocionar productos, es un sistema de comunicación cara a cara, desarrollado por personas naturales, llamadas visitadores médicos.

El éxito de cualquier visita médica es conseguir que el médico formule o recomiende los productos ofertados por el laboratorio farmacéutico que representa.

Aprovechando que las organizaciones farmacéuticas utilizan el correo electrónico (e-mail) como medio para informar, promocionar y divulgar sus productos y servicios, surge la posibilidad de potenciar esta alternativa a través de un visitador medico virtual que facilite a la comunidad médica el acceso al conocimiento y la apropiación de nuevos productos, así como la actualización de los existentes.

Para dar a conocer sus productos y mantener la fidelidad de los médicos, los laboratorios entregan información oportuna sobre los cambios y los adelantos científicos dentro del campo farmacéutico (Thompson, S. 2009).

Los grandes adelantos en materia de tecnología y de las nuevas concepciones en torno al ejercicio de la comercialización a escala mundial, han llevado a que se exploten y se aprovechen al máximo estas posibilidades proyectadas al servicio de la promoción y la negociación de productos y servicios; se origina así la base para el surgimiento y la potenciación del e-commerce como medio y estrategia para facilitar el flujo operacional en los mercados, así como la fluidez y la eficiencia en la comunicación, tanto con los proveedores como con los clientes y los beneficiarios, sin que la ubicación espacial y temporal se constituya en obstáculo para ello, y se permita el acceso a más información.

Dentro de este campo, los laboratorios productores de medicamentos y generadores de conocimientos científicos también quedan inmersos, pues, como toda empresa, su accionar se encamina a la difusión pertinente y oportuna para atraer las demandas, pero con la novedad de recurrir a un sistema que facilita el contacto permanente con los clientes, en aras de asegurar el posicionamiento en el mercado y la apertura de nuevos segmentos a los cuales se accede de forma interactiva sirviéndose de las bases de datos.

Esta dinámica en el campo de la comercialización, es la que se conoce como el B2C, y alude a la utilización de la Internet como herramienta para llegar de forma directa al consumidor cuando este visita el sitio web de una empresa o industria, donde, además de encontrar información, también puede llegar a adquirir un bien o un servicio a bajo costo, o, del mismo modo, realizar cualquier tipo de transacción comercial (Porter, 2001).
La clave de la visita médica virtual es la integración interna de los conceptos que conforman el e-commerce, y la integración con otros canales fuera de la empresa, que permita construir un modelo que utilice la innovación para enlazar, sincronizar el proceso, y que, a la vez, lo haga eficaz (Kalakota, 2003).

En un artículo publicado por Scrip Magazine en 1997, de Devin y Hemsley, se estima que la industria farmacéutica gasta, aproximadamente, el $35 \%$ de sus ingresos en actividades de mercadeo.

La propuesta como tema de investigación: Visitador médico virtual una ventana estratégica para el desarrollo del mercado farmacéutico, direccionada dentro del área de investigación Estado, Empresa, Globalización y Desarrollo, considerada, a su vez, dentro del contexto de la línea de investigación e-commerce; compra y venta de bienes y servicios en Internet, se desarrolló desde la relación médico-laboratorio en lo concerniente a las actualizaciones científicas promovidas para que los médicos, a través del visitador médico virtual, se apropien de los conocimientos en torno a los productos ofertados, para que sean formulados y recomendados en su práctica profesional. Con tal perspectiva, esta investigación se basó en la siguiente pregunta: ¿Cuáles son las características del servicio de visita médica virtual que esperan los médicos?

Para su desarrollo, se trazó como objetivo general: describir las características que esperan los médicos de un servicio de visita médica virtual. A partir de aquí, se generaron los siguientes objetivos específicos:

- Detallar la percepción de los médicos acerca de la adopción del comercio electrónico en la visita médica.

- Describir las formas como los médicos esperan que los laboratorios farmacéuticos promocionen virtualmente sus medicamentos.

- Pormenorizar las formas como los médicos esperan que los laboratorios farmacéuticos contribuyan a la actualización en sus especialidades.

\section{Métodos}

La investigación propuesta es una investigación cuantitativa-descriptiva-exploratoria. Cuantitativa porque permitió examinar los datos de manera numérica; especialmente, en el campo de la estadística. Descriptiva porque busca describir las características del fenómeno. Y exploratoria porque es una primera aproximación al fenómeno.

El estudio corresponde a un diseño de tipo descriptivoexploratorio-transversal.

Los estudios descriptivos buscan especificar las propiedades, las características y los perfiles de las personas, 
los grupos o las comunidades, los procesos, los objetos o cualquier otro fenómeno que se someta a un análisis (Danke, 1989).

En el estudio descriptivo se miden, se evalúan o se recolectan datos sobre diversos conceptos, aspectos, dimensiones o componentes del fenómeno investigado. Es exploratorio porque se realiza cuando el objetivo es examinar un tema o problema poco estudiado que no se ha abordado antes. En este caso, la oferta de un servicio de visita médica virtual. Es, además, un estudio de carácter transversal, porque su propósito es describir variables y analizar su incidencia y su interrelación en un momento dado.

Para evaluar las características que se investigarán, se utilizó la encuesta como técnica o instrumento, aplicada de manera individual a facultativos de Barranquilla y Riohacha. Este instrumento estuvo caracterizado mediante las siguientes variables: actualización de los servicios virtuales prestados por la visita médica; capacitación en el uso de las plataformas utilizadas, y prestación óptima de los servicios ofrecidos en relación con medicamentos de alta calidad.

Para efectos de imprimir organización al trabajo investigativo, se definieron cuatro fases puntuales, así:

- Fase 1. Definición de la situación problema: exploración de la situación; diseño del Instrumento.

- Fase 2. Trabajo de campo: recolección de datos y organización de la información.
- Fase 3. Análisis de la información: descripción de los resultados; evaluación y análisis de resultados.

- Fase 4. Conclusiones: ante el desconocimiento del tamaño exacto de la población, fue necesario trabajar con la siguiente fórmula:

$\mathrm{n}=\left[\mathrm{Z}^{2} \mathrm{c}^{*} \mathrm{p}^{*} \mathrm{q}\right] /\left[\mathrm{E}^{2}\right]$

Donde: $n=$ tamaño de la muestra, y $Z c=$ nivel de confianza requerido para generalizar los resultados.

El nivel de confianza se consideró en el 95\%, que correspondió a 1,92. p.q=variabilidad del fenómeno estudiado $p=0,5$ y $q=0,5 ; E=$ error máximo permitido se ubica el 0,02 y el 0,10 y se decidió tomar el valor de 0,06.

Al remplazar en la formula, se obtuvo:

$n=\left[1,92^{\star} 0,5^{\star} 0,5\right] /\left[0,06^{\star} 0,06\right]$

$n=[0,48] /[0,0036]$

$n=133$

El tamaño de la muestra fue de 133 médicos, focalizados en Barranquilla y Riohacha, en la Costa Atlántica colombiana.

\section{Resultados y Conclusiones}

Al analizar la Gráfica 1 (¿Cuánto tiempo lleva utilizando la visita médica?) se puede apreciar que de los 133 médicos se determinó que 118, quienes representan el $88 \%$ de la muestra, tienen más de un año de estar recibiendo la visita médica; mientras, el $12 \%$ tiene menos de un año.

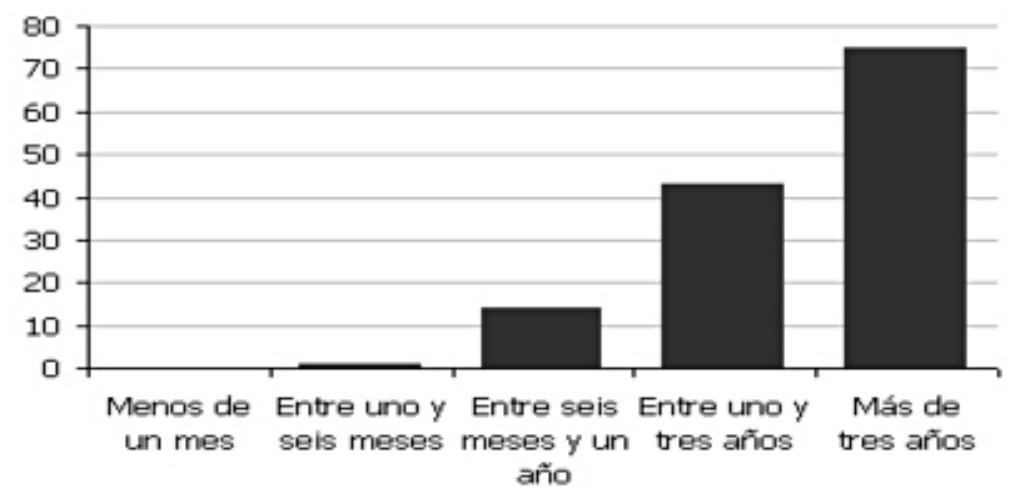

Gráfica 1. ¿Cuánto tiempo lleva utilizando la visita médica?

La Gráfica 2 registra que en relación con la frecuencia con que recibe a los visitadores médicos, 99 de los médicos, equivalentes al $75 \%$, manifestaron recibir visitas médicas más de una vez a la semana; el $7 \%$ restante la recibe ocasionalmente, y el $18 \%$, menos de una vez al mes. 


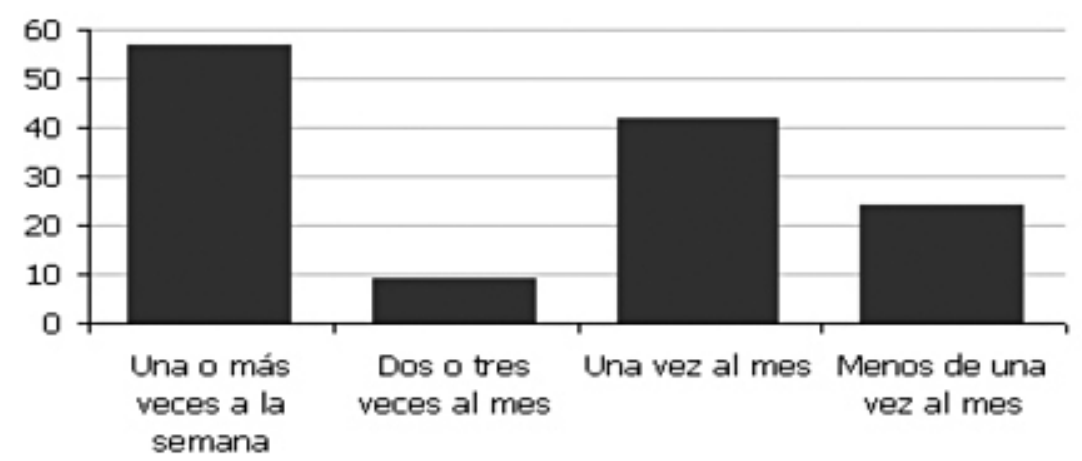

Gráfica 2 ¿Con qué frecuencia recibe a visitadores médicos?

El análisis del uso del servicio de la visita médica, permitió concluir que la mayor parte de los médicos cuentan con el servicio permanente de un visitador.

En la Gráfica 3 se evidencia que, con respecto a la satisfacción general de la visita médica, el 89\% de los médicos encuestados declararon estar satisfechos con la visita médica. Una minoría, representada en el 11\%, manifestó insatisfacción.

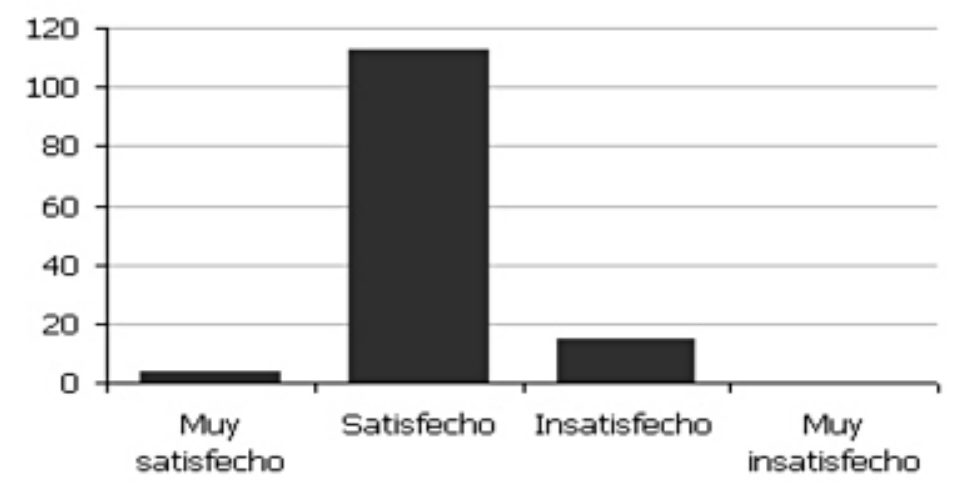

Gráfica 3. ¿Cuál es su grado de satisfacción general con la visita médica?

Ante la solicitud de la pregunta: En comparación con otras alternativas de promoción, como videos, la visita médica es..., la Gráfica 4 identifica cómo el $86 \%$ de los encuestados señaló que sería mucho mejor recurrir a estrategias como los videos, y el $14 \%$ restante no estuvo de acuerdo con ello.

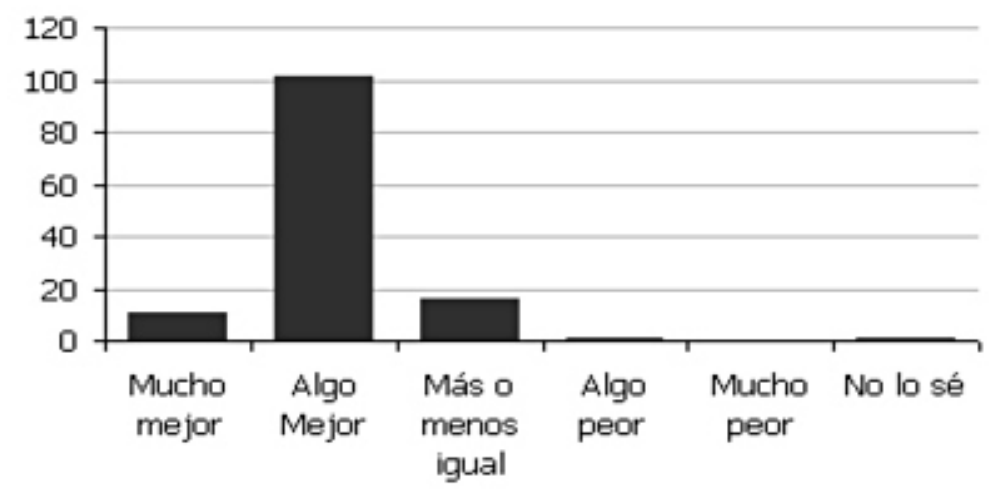

Gráfica 4. En comparación con otras alternativas de promoción, como videos, la visita médica es... 
En la Gráfica 5, alrededor de las características de la visita médica, el 51\% de los médicos expresaron que para ellos lo más importante es el conocimiento del producto, y que la visita sea breve; el 14\%, por su parte, indicó que espera la cancelación del valor de una consulta por cada visita; el 11\% señaló como importante recibir muestras de medicamentos; el 10\% optó por la actualización permanente de la información; el 7\% valoró más la eficacia de la visita, en tanto que el 4\% indicó que lo más importante es la visita oportuna. El 3\% señaló otras opciones.
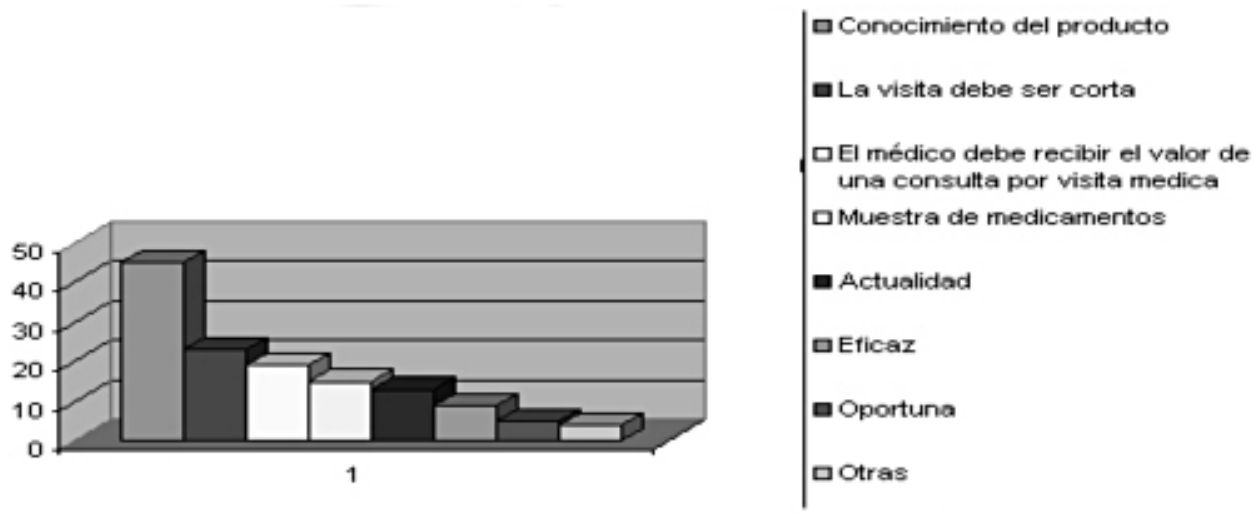

\section{Grafica 5. Escriba en orden de importancia, empezando por la No. 1 como la más importante, las características de la visita médica}

En cuanto al género del visitador médico que llega al consultorio, el 95\% de los médicos mostró indiferencia ante el género, y esto se manifiesta en la Gráfica 6.

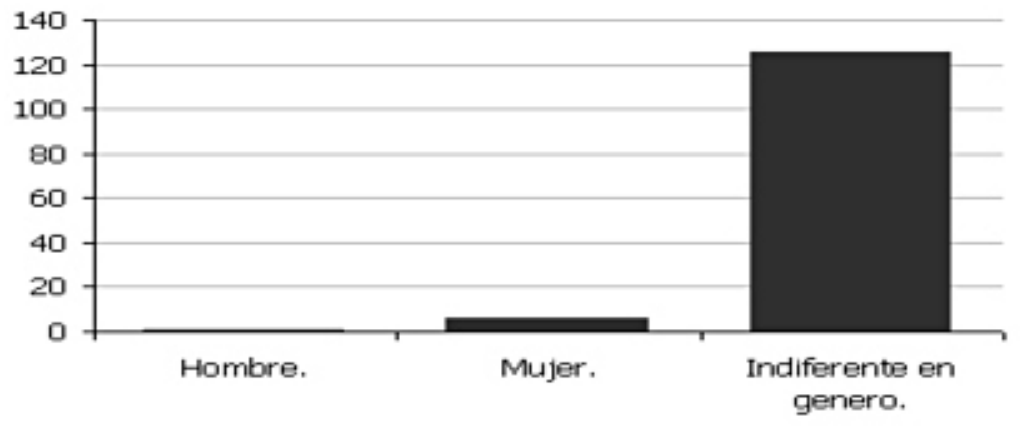

Gráfica 6. Prefiere ser atendido en la visita médica por...

Se concluyó que en cuanto a satisfacción general, para más del $50 \%$ de los médicos lo más importante es: el conocimiento del producto, la brevedad de la visita, su eficacia, así como la actualización de la información. Mientras, es indiferente el género de quien los atienda.

Sobre la utilización para la consulta de los productos promocionados registrados en la Gráfica 7, el 95\% de los médicos consideró válida la Internet como medio para consultar y ampliar sus conocimientos sobre los medicamentos ofertados. El 5\% restante se mostró dudoso ante dicha alternativa. 


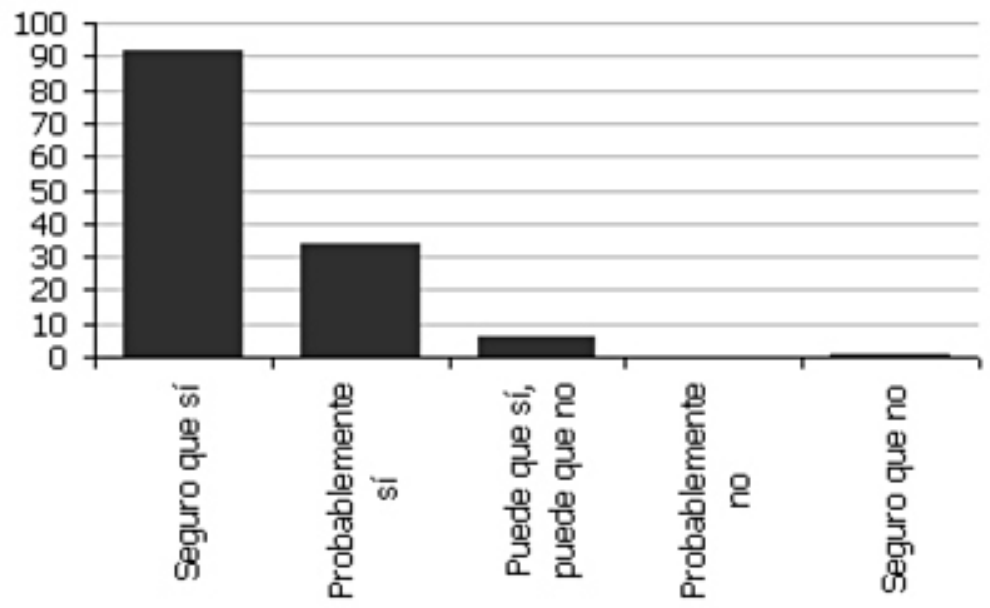

Gráfica 7. ¿Utilizaría usted la Internet para consultar los productos ofertados durante la visita médica?

Ante la propuesta de uso de la Internet como medio de información que reemplace la visita médica física, representada en la Gráfica 8, el $81 \%$ de los médicos encuestados se mostraron partidarios de su utilización, y valoraron la efectividad y la actualización de la información; el 19\% se mostró en desacuerdo. En efecto, el 93\% expresó su agrado ante la idea de que un visitador médico le atendiera por medio de un video chat. Solo un 7\% expresó oposición.

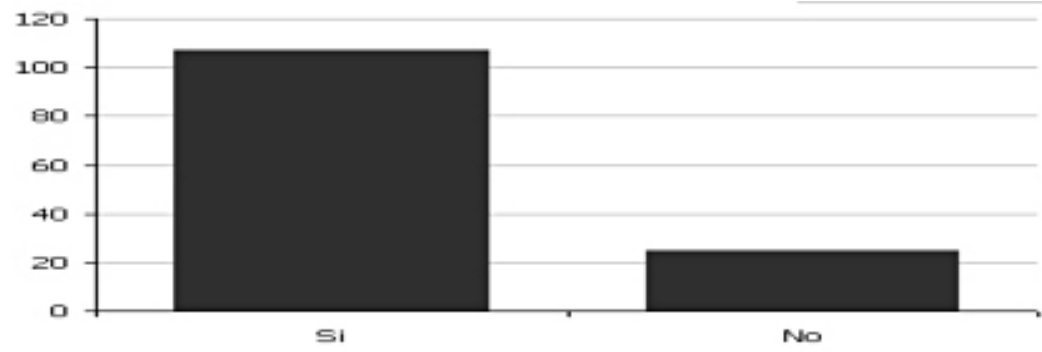

Gráfica 8. ¿Le gustaría recibir informes y atenciones a través de Internet, que remplacen la visita médica física?

En este mismo sentido, y según la Gráfica 9 , el $63 \%$ de los médicos consideró que un ambiente virtual con variadas opciones sobre el tema puede llegar a prestar una mejor atención que la que tradicionalmente se viene haciendo en la visita física. El 37\% no tuvo la misma opinión.

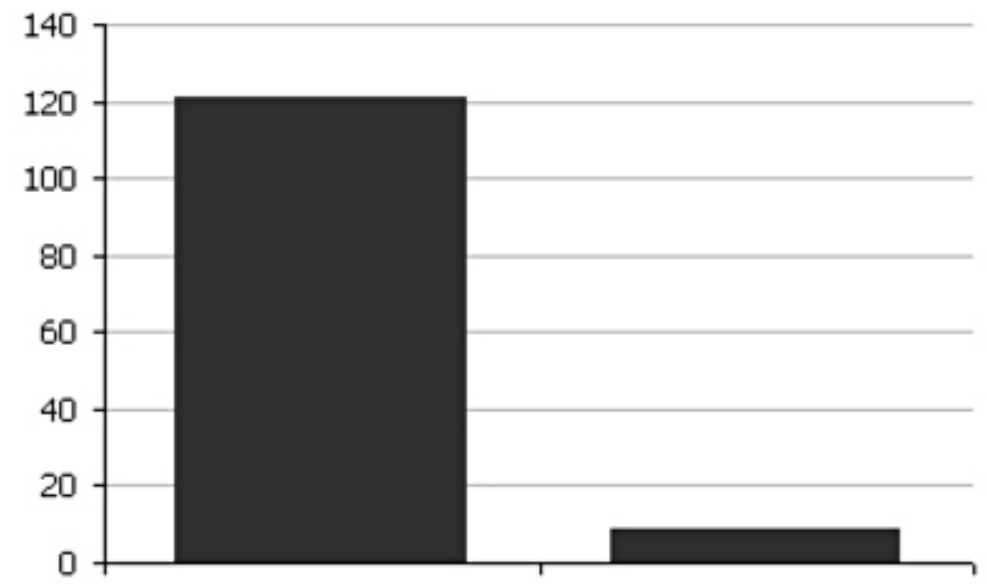

$\mathrm{Si}$

No

Gráfica 9. ¿Le agradaría que un visitador médico le atendiera virtualmente por video chat? 
Respecto a la recomendación de una visita médica a otros colegas, la Gráfica 10 muestra que el 95\% de los facultativos señaló haberlo hecho, y el $5 \%$, no; ello permitió deducir que la promoción de la visita médica virtual también podría recomendarse dentro del gremio.

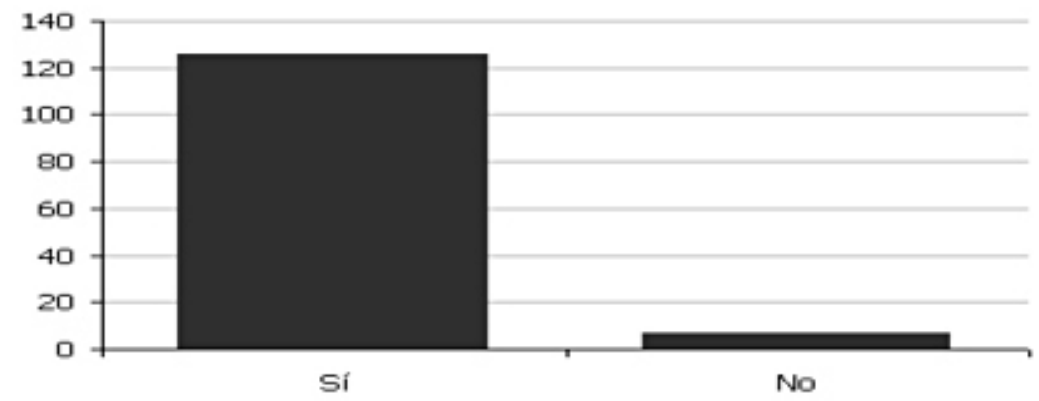

\section{Gráfica 10. ¿Ha recomendado usted a otros colegas una visita médica?}

Sobre la intención de uso y las recomendaciones analizadas en la encuesta, se concluyó que existe una marcada inclinación y agrado hacia el uso de las nuevas tecnologías de la información para acceder al conocimiento de productos y servicios ofertados en las visitas médicas tradicionales.

En lo referente a la satisfacción de atributos registrados en la Gráfica 11, vale mencionar que para el $99 \%$ de los médicos encuestados la interrelación personal es importante en cuanto a la visita médica. De igual manera, el 100\% estuvo de acuerdo en que es importante para la actualización del conocimiento de lo ofertado. Así también, el manejo de la literatura médica obtuvo un 100\% de importancia en relación con los medicamentos; de allí que el $98 \%$ de los encuestados indicaran que es esencial tener la muestra física del producto ofrecido es al momento de la visita. Para el $97 \%$ de los médicos es muy importante la periodicidad de las visitas, para el conocimiento, el manejo y la obtención de las muestras médicas.

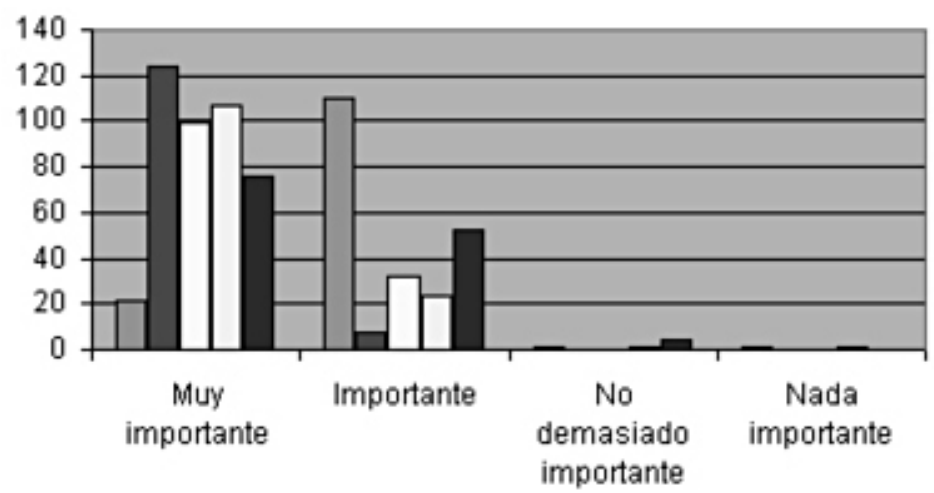

\section{Gráfica 11. ¿Qué grado de importancia le da usted a los siguientes aspectos a la hora de la visita médica?}

Estos resultados llevaron a pensar que la implementación de la visita médica virtual sería eficiente en un alto porcentaje, pues permitiría la interrelación con personal especializado, y ampliaría y daría permanencia a la actualización del conocimiento y al acceso a literatura médica vigente. A través de dichas estrategias virtuales, el médico tendría la facilidad para optar por los medicamentos ofrecidos, los cuales podría recibir físicamente por medio de mensajería. 
En lo referente a la Gráfica 12 (valoración del servicio de la visita médica), el $72 \%$ del total de los encuestados indicó que la visita médica cubre las necesidades de información y conocimiento de productos; el $25 \%$ se mostró en desacuerdo con ellos, y al $2 \%$ restante no le interesa.
Sobre la incomodidad de la visita médica, el 50\% afirmó que el hecho no se la genera. El 35\% dio a entender que sí le resulta incomoda, debido a que los visitadores llegan en horarios de consulta y de atención a los pacientes, y en la mayoría de los casos es prolongada. El $15 \%$ restante es indiferente a esto.

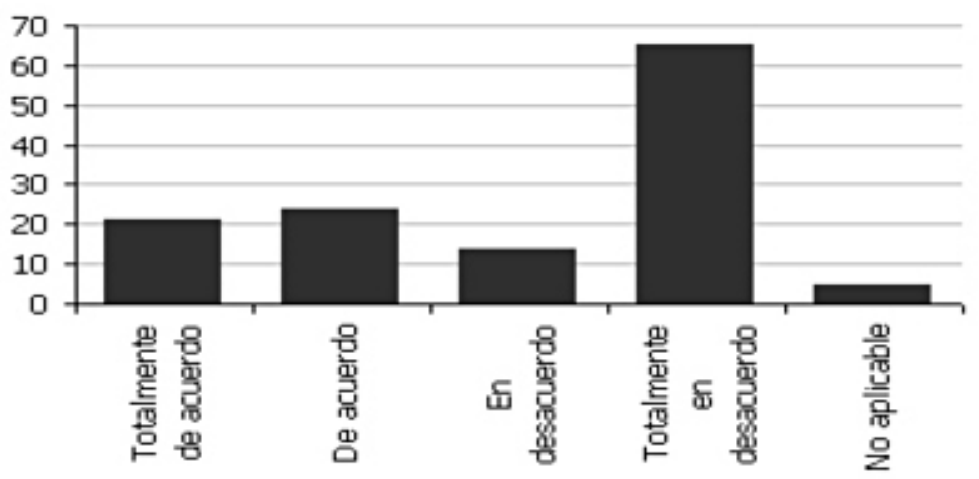

Gráfica 12. ¿La visita médica le genera incomodidad?

Sobre la oferta de un mismo producto por parte de diferentes laboratorios (Gráfica 13), el 94\% dijo estar de acuerdo, pues ello les permite contar con varias opciones para la formulación, y el 6\% estuvo en desacuerdo.

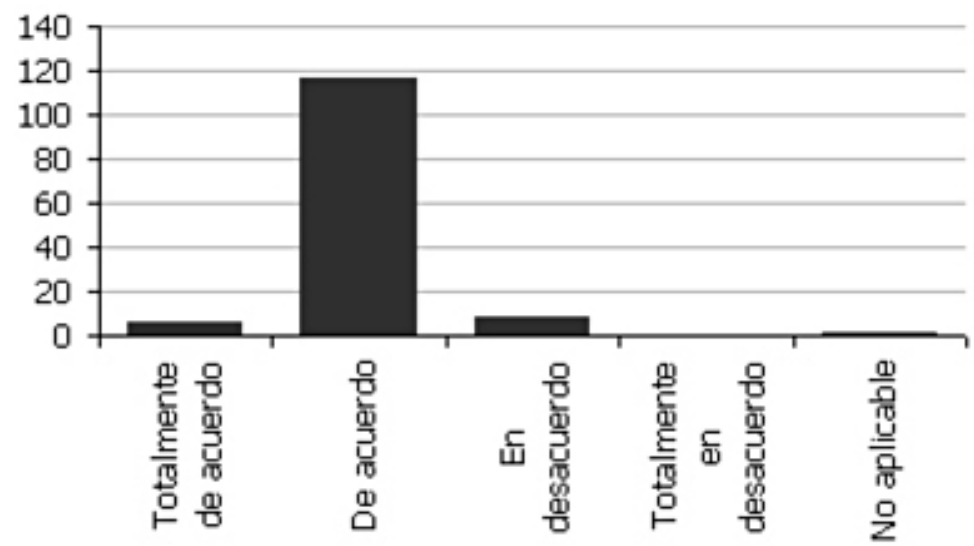

Gráfica 13. ¿La visita médica de diferentes laboratorios por el mismo producto le facilita la formulación?

Respecto de la valoración del servicio de visita médica, se evidencia que aun cuando es positivo el valor que se le da, y, a la vez, amplía la gama en cuanto a oferta de productos de distintos laboratorios, hay incomodidad por el horario de visita y el tiempo de diálogo con el médico. Esto último permitió concluir que la posibilidad de ofrecer el visitador medico virtual evitaría dichos inconvenientes sin perder la esencia ni los objetivos de este servicio.

En torno a las recomendaciones y las sugerencias que los médicos encuestados dieron sobre la experiencia de la visita médica, el 95\% indicó que sería probable recurrir a otra estrategia para acceder al mismo servicio; el 5 $\%$ restante no lo vio como probable.
La Gráfica 14, producto de la encuesta, registra que el $92 \%$ de los participantes aseguró no haber tenido problema alguno a la hora de recibir la visita médica, y el $8 \%$ afirmó haberlo tenido. 


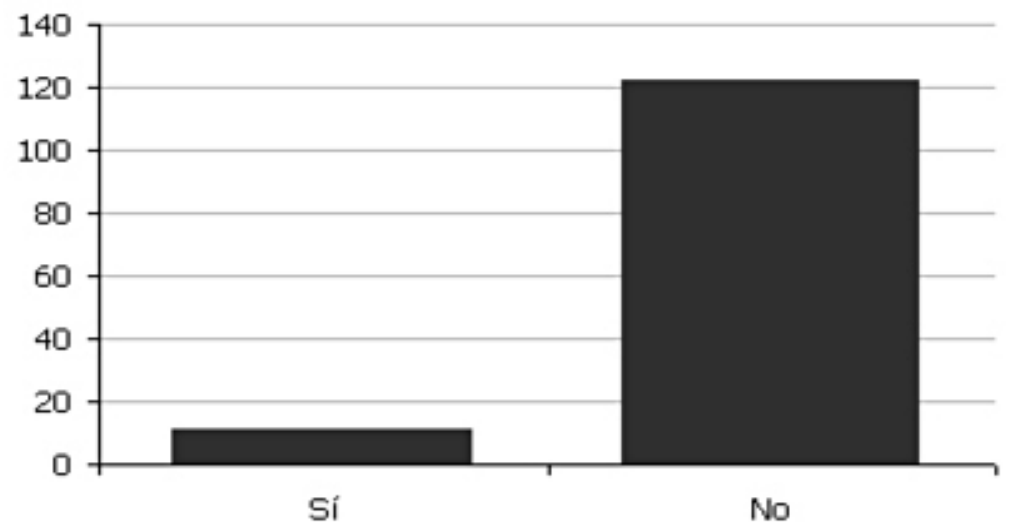

Gráfica 14. ¿Ha tenido usted algún problema a la hora de recibir o usar la visita médica?

En lo referente a las recomendaciones y las sugerencias por parte de la muestra encuestada, se concluyó que es altamente probable la recurrencia a la implementación de la visita médica virtual como estrategia para la promoción y el desarrollo del mercado farmacéutico.

\section{Referencias bibliográficas}

Büscher, R. (2003). Europa ante el desafío del negocio electrónico. VII Jornada EUROCOM-2BDigital: Internet como una herramienta de negocio en la empresa (2002: Barcelona) Recuperado de http://www.uoc.edu/symposia/euroecom/esp/art/buescher0203/buescher0203.html

Campinelli, A. (2007). Comercio electrónico. Recuperado de http://www.monografias.com/trabajos12/monogrr/monogrr.shtml

Dankhe, G. L. (1989). Investigación y comunicación, en C. Fernández-Collado y G. L. Dankhe (comps.). La Comunicación Humana: Ciencia Social. México: Ed. McGrawHill.

Hernández Sampieri, R., Fernández Collado, C. \& Baptista, P. (2007). Metodología de la investigación (4ª ${ }^{\text {. ed.). }}$. México: McGraw Hill/Interamericana editores, S.A. de C.V.

Homs, C. (2003). La tecnología de la información: herramienta esencial para gestionar la productividad. En VII
Jornada EUROCOM-2BDigital: Internet como una herramienta de negocio en la empresa (2002: Barcelona). Recuperado de http://www.uoc.edu/symposia/euroecom/ esp/art/homs0203/homs0203.html>

Kalokata, R. (2003). Digitalización de procesos en las pymes: de la visión a la ejecución. En VII Jornada EUROCOM-2BDigital: Internet como una herramienta de negocio en la empresa (2002: Barcelona). Recuperado de http://www.uoc.edu/symposia/euroecom/esp/art/kalakota0203/kalakota0203.html

Porter, M. (2001). La estrategia de del internet, Harward Busines Review. Recuperado de http://www.docstoc. com/docs/1190689/Michael-Porter---Internet-y-la-estrategia---gestión

Thompson, S. (2009). Manual del visitador médico para mercados de alta competencia. Recuperado de http:// www.promofar.com/articulos/i-definicion-visitador-medico.html 